\title{
FROBENIUS CALCULATIONS OF PICARD GROUPS AND THE BIRCH-TATE-SWINNERTON DYER CONJECTURE
}

\author{
BY \\ RAYMOND T. HOOBLER( $\left.{ }^{\mathbf{1}}\right)$
}

\begin{abstract}
Let $Y \subset \mathrm{P}^{m}$ be a subvariety of codimension $d$ defined by an ideal $I$ in char $p>0$ with $H^{1}(Y, O(-1))=0$. If $t$ is an integer greater than $\log _{p}(d)$ and $H^{i}\left(Y, I^{n} / I^{n+1}\right)=0$ for $n \gg 0$ and $i=1,2$, then $\operatorname{Pic}(Y)$ is an extension of a finite $p$-primary group of exponent at most $p^{t}$ by $Z[O(1)]$ and $\mathrm{Br}^{\prime}(Y)(p)$ is a group of exponent at most $p^{t}$. If $Y$ is also smooth and defined over a finite field with $\operatorname{dim} Y$ $\langle p$ and $p \neq 2$, then the B-T-SD conjecture holds for cycles of codimension 1. These results are proved by studying the etale cohomology of the Frobenius neighborhoods of $Y$ in $\mathbf{P}^{m}$.
\end{abstract}

Let $i: Y \rightarrow \mathrm{P}^{m}$ be a smooth subvariety of $\mathrm{P}^{m}$ over C. If $\operatorname{dim}(Y) \geqslant$ $1 / 2(m+2)$, then Barth and Larsen have shown that $\operatorname{Pic}(Y)=Z$ and it is generated by $O_{Y}(1)$. This follows easily from their isomorphism theorems for the cohomology of smooth subvarieties of low codimension and the exponential sequence. Ogus has given an algebraic proof of this result in characteristic 0 by studying the cohomology of the formal completion $Y$ of $\mathrm{P}^{m}$ along $Y$ and using the exponential map to pass from $\operatorname{Pic}(y)$ to $\operatorname{Pic}(Y)$.

We use the same approach via $Y$, but the techniques required to study $\operatorname{Pic}(Y)$ are quite different in char $p>0$. Clearly a general exponential map does not exist. Instead we have the Frobenius map. Moreover if $Y_{0} \subset Y$ is a square zero deformation, then the truncated exponential sequence shows that $\operatorname{Pic}(Y) \rightarrow$ $\operatorname{Pic}\left(Y_{0}\right)$ has a $p$-torsion kernel and cokernel. In particular if $i: Y \rightarrow \mathrm{P}^{m}$ is a closed embedding defined by an ideal $I$ and $Y$ is the formal scheme obtained by completing $\mathrm{P}^{m}$ along $Y$, then $\operatorname{Pic}\left(Y_{N}\right)$ has the same rank for any $N$ but $\operatorname{Pic}(Y)$ may have smaller rank. This is essentially due to a $p$-adic limit phenomenon wherein the limit may be zero without the groups being 0 . By requiring that $H^{i}\left(Y, I^{n} / I^{n+1}\right)=0$ for $i=1,2$ and $H^{1}\left(Y, O_{Y}(-1)\right)=0$, we can avoid this and can obtain a remarkably strong theorem-Pic $(Y)$ has rank 1 and contains no $p^{n}$ torsion for $n>\log _{p}(d)$ where $d=\operatorname{codim}\left(Y, \mathrm{P}^{m}\right)$. Moreover we verify the BirchTate-Swinnerton Dyer conjectures in codimension 1 for such smooth varieties

Received by the editors March 31, 1975.

AMS (MOS) subject classifications (1970). Primary 14C20, 14Fxx.

Key words and phrases. Frobenius neighborhood, Brauer group.

(1)Partially supported by NSF grant GP 43002. 
over a finite field by using a recent result of Bloch which interprets fppf cohomology in terms of crystalline cohomology. The strength of this theorem shows that the hypotheses on $I^{n} / I^{n+1}$ are not usually satisfied. However in char 0 Serre duality implies $H^{i}\left(Y, I^{n} / I^{n+1}\right)=0$ for $n \gg 0$ and $i \neq \operatorname{dim} Y$ since $Y$ has an ample normal bundle (see remarks following the theorem). Our use of the Frobenius map will help explain why this fails in char $p>0$ and why the analytical flavor of algebraic geometry in char 0 must be replaced by a $p$-adic flavor in char $p$ even when the questions are formulated in the same way.

From now on $k$ will denote a field of characteristic $p>0$. We will be computing cohomology groups in various topologies on $X$ such as in $X_{\mathrm{pl}}$, the faithfully flat, locally of finite presentation site, and in $X_{\mathrm{Zar}}$, the Zariski site. If no subscript is indicated, we are working in the Zariski site. We will use without mention the isomorphism $H^{i}\left(X_{\mathrm{pl}}, F\right) \cong H^{i}(X, F)$ for a coherent sheaf $F$ on $X$ where $F$ is a sheaf on $X_{\mathrm{pl}}$ via $\Gamma(T, F)=H^{0}\left(T, p^{*} F\right)$ for $p: T \rightarrow X$ in $X_{\mathrm{pl}}$.

Let $i: Y \rightarrow \mathrm{P}^{m}$ be a closed embedding defined by the ideal $I . i_{N}: Y_{N} \rightarrow \mathrm{P}^{m}$ will denote the closed embedding of the scheme defined by $I^{N+1}$ in $\mathbf{P}^{m}$. $i_{(r)}: Y_{(r)} \rightarrow \mathrm{P}^{m}$, the $r$ th Frobenius neighborhood of $Y$ in $\mathrm{P}^{m}$, will denote the closed embedding defined by $I^{(r)}$ where $I^{(r)}$ is the ideal in $O_{\mathrm{p} m}$ generated by $p^{r}$ th powers of elements in $I$. Thus we have a cartesian diagram

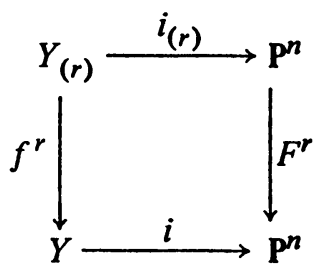

where $F^{r}$ is the $r$ fold composite of $F=F_{\mathbf{p} m}$, the Frobenius map of $\mathbf{P}^{m}$. For simplicity of notation we will write $f$ for the restriction of $F$ to $Y_{(r)}$. The context will always make clear which Frobenius neighborhood of $Y$ we are using.

Our main tool will be a result in [3] which we describe here for the case $i: Y \rightarrow \mathrm{P}^{m}$ in order to fix notation. Let $\Omega^{r}=\Omega_{\mathrm{P} m / k}^{r}$ and $Z^{r}, B^{r}$ be the sheaves of abelian groups of closed, exact $r$ forms on $\mathbf{P}^{m}$ respectively. They define coherent sheaves on $\mathbf{P}^{m}$ when they are regarded as subsheaves of $F_{*} \Omega^{r}$. Now $F: \mathrm{P}^{m} \rightarrow \mathrm{P}^{m}$ is a purely inseparable Galois covering. The fundamental exact sequence on $\mathbf{P}_{\mathrm{pl}}^{m}$ of [3] is

$$
0 \rightarrow \mathrm{G}_{m, \mathrm{P} m} \rightarrow F_{*} \mathrm{G}_{m, \mathrm{P} m} \stackrel{d \ln }{\longrightarrow} Z^{1} \stackrel{C-I}{\longrightarrow} \Omega^{1} \rightarrow 0
$$

where $C: Z^{1} \rightarrow \Omega^{1}$ is the Cartier operator and $I: Z^{1} \rightarrow \Omega^{1}$ is the "inclusion." Since we are using the absolute Frobenius on $\mathrm{P}^{m}$, the map $\psi$ appearing in [3] is the identity. Note that $C$ is a linear map while $I$ is only $p$-linear since $Z^{1}$ is being regarded as a subsheaf of $F_{*} \Omega^{1}$. 
Our first result is the basic tool for analyzing the fppf cohomology of Frobenius neighborhoods of $Y$.

Proposition 1. Let $i: Y \rightarrow \mathbf{P}_{k}^{m}$ be a subscheme of $\mathbf{P}^{m}$. There are exact sequences in $\widetilde{Y}_{\mathrm{pl}}$ :

$$
\begin{gathered}
0 \rightarrow \mathrm{G}_{m, Y} \rightarrow f_{*} \mathrm{G}_{m, Y_{(1)}} \rightarrow i^{*} Z^{1} \stackrel{c-i}{\rightarrow} i^{*} \Omega^{1} \\
0 \rightarrow i^{*} Z^{1} \rightarrow f_{*} i_{(1)}^{*} \Omega^{1} \rightarrow f_{*} i_{(1)}^{*} \Omega^{2}
\end{gathered}
$$

where $c, i$ denote the restrictions of $C, I$ to $Y_{\mathrm{pl}}$ and $i^{*} Z^{1}$, etc. are the coherent sheaf restrictions of $Z^{1}$, etc. which are then regarded as fppf sheaves on $Y$.

Proof. Since $F: \mathbf{P}^{m} \rightarrow \mathbf{P}^{m}$ is a purely inseparable Galois covering, so is $f: Y_{(1)} \rightarrow Y[3]$. Hence there is an exact sequence in $\widetilde{Y}_{\mathrm{pl}}$,

$$
0 \rightarrow \mathbf{G}_{m, y} \rightarrow f_{*} \mathbf{G}_{m, y_{(1)}} \rightarrow Z_{Y_{(1)} / Y}^{1} \stackrel{c-i}{\longrightarrow} \psi^{*} \Omega_{Y_{(1)} / Y}^{1} \rightarrow 0,
$$

where $\psi \circ f=F_{Y}$ is the Frobenius map on $Y, c$ is the Cartier operator on $Y, i$ is the "inclusion" map on $Y$, and $Z_{Y_{(1)} / Y}^{1}$ is defined by the exact sequence

$$
0 \rightarrow Z_{Y_{(1)} / Y}^{1} \rightarrow f_{*} \Omega_{Y_{(1)} / Y}^{1} \rightarrow f_{*} \Omega_{Y_{(1)} / Y}^{2}
$$

But $\Omega_{Y(1) / Y}^{i}=i_{(1)}^{*} \Omega_{\mathrm{P} m}^{i}$ for $i=1,2$ and so $Z_{Y_{(1)} / Y}^{1} \simeq i^{*} Z^{1}$ as coherent sheaves on $Y$. Moreover $\psi: Y \rightarrow Y_{(1)}$ is the closed embedding defined by $I / I^{(1)}$. Since $i_{(1)} \psi=i, \psi^{*} \Omega_{Y(1)}^{1} Y_{Y}=i^{*} \Omega^{1}$. Once these identifications have been made, we find that $C, I$ are identified with the restriction to $Y$ of the corresponding operators on $\mathrm{P}^{m}$ which accounts for the notation $c, i$.

In order to describe the effect of $f^{*}$ on $\operatorname{Pic}\left(Y_{(n)}\right)$ and

$$
\operatorname{Br}^{\prime}\left(Y_{(n)}\right)=H^{2}\left(Y_{(n) \mathrm{p} 1}, \mathbf{G}_{m}\right) \text {, }
$$

the cohomological Brauer group of $Y_{(n)}$, in terms of the Zariski cohomology of $i_{(n)}^{*} Z^{1}$ and $i_{(n)}^{*} \Omega^{1}$, we need an inductive formulation provided by the next result.

Proposition 2. Let $i: Y \rightarrow \mathrm{P}^{m}$ be a closed subscheme with tautological bundle $O_{Y}(1)=O(1)$ defined over a field $k$.

(a) If $H^{0}\left(Y, O_{Y}\right)=k$, then $f^{*}: \operatorname{Pic}(Y) \rightarrow \operatorname{Pic}\left(Y_{(1)}\right)$ is injective and $H^{0}\left(Y_{(1)}, O_{Y_{(1)}}\right)=k$.

(b) If $H^{0}\left(Y, O_{Y}\right)=k, H^{1}\left(Y, O_{Y}(-1)\right)=0$, then there is an exact sequence

$$
0 \rightarrow \operatorname{Pic}(Y) \rightarrow \operatorname{Pic}\left(Y_{(1)}\right) \rightarrow Z / p Z \rightarrow 0
$$

where the image of $\mathrm{O}_{Y_{(1)}}(1)$ is a basis element of $Z / p Z$ and $f^{*}: \operatorname{Br}^{\prime}(Y) \rightarrow$ $\operatorname{Br}^{\prime}\left(Y_{(1)}\right)$ is injective. Moreover $H^{1}\left(Y, i^{*} \Omega^{1}\right)=k$ and $H^{1}\left(Y_{(1)}, O_{Y_{(1)}}(-1)\right)=0$. in $\widetilde{Y}_{\mathrm{pl}}$,

Proof. In addition to (1.1) and (1.2), we need three other exact sequences 


$$
\begin{gathered}
0 \rightarrow i^{*} \Omega^{1} \rightarrow \bigoplus_{1}^{m+1} O_{Y}(-1) \rightarrow 0_{Y} \rightarrow 0, \\
0 \rightarrow i^{*} B^{1} \rightarrow i^{*} Z^{1} \stackrel{c}{\rightarrow} i^{*} \Omega^{1} \rightarrow 0, \\
0 \rightarrow 0_{Y} \rightarrow f_{*} 0_{Y_{(1)}} \rightarrow i^{*} B^{1} \rightarrow 0
\end{gathered}
$$

which are obtained by restriction just as above. Moreover $i^{*} B^{1}=\bigoplus_{1}^{s} O_{Y}\left(-n_{i}\right)$ where $n_{i}>0$ since $F_{*} O_{\mathrm{p} m}=\bigoplus_{0}^{s} O\left(-n_{i}\right)$ where $n_{0}=0$ [2, Chapter III, Corollary 6.4].

(a) Let $B_{m}^{1}$ be the cokernel of $\mathrm{G}_{m, Y} \rightarrow f_{*} \mathbf{G}_{m, Y_{(1)}}$. The hypothesis on $Y$ shows that $H^{0}(Y, O(-1))=0$ and so $H^{0}\left(Y, i^{*} \Omega^{1}\right)=0=H^{0}\left(Y, i^{*} Z^{1}\right)$. Thus $H^{0}\left(Y, B_{m}^{1}\right)=0$ as desired. Moreover (1.5) shows that $H^{0}\left(Y_{(1)}, O_{Y_{(1)}}\right)=k$.

(b) The hypothesis and (1.3) shows that $H^{0}\left(Y, O_{Y}\right) \rightarrow H^{1}\left(Y, i^{*} \Omega^{1}\right)$ is an isomorphism. Since the first deRham Chern class of $O_{Y_{(1)}}(1)$ lies in the image of $H^{1}\left(Y, i^{*} Z^{1}\right), H^{1}\left(Y, i^{*} Z^{1}\right)=k$. Now $i^{*}$ is $p$-linear and so the kernel of $c^{*}-i^{*}=Z / p Z$. Since $H^{0}\left(Y, i^{*} \Omega^{1}\right)=0$, this shows that $H^{1}\left(Y, B_{m}^{1}\right)=Z / p Z$ and there are exact sequences

$$
0 \rightarrow \operatorname{Pic}(Y) \rightarrow \operatorname{Pic}\left(Y_{(1)}\right) \rightarrow Z / p Z \rightarrow 0, \quad 0 \rightarrow \operatorname{Br}^{\prime}(Y) \rightarrow \operatorname{Br}^{\prime}\left(Y_{(1)}\right)
$$

Finally since $F_{*} O(-1)=\bigoplus O\left(-m_{i}\right), m_{i}>0, f_{*} O_{Y_{(1)}}(-1)$ is a sum of $O_{Y}\left(-m_{i}\right)$. Hence $H^{1}\left(Y_{(1)}, O_{Y_{(1)}}(-1)\right)=0$.

Our main result now follows by comparing the action of $f$ and $F_{Y}$ on $H^{i}\left(Y_{\mathrm{pl}}, \mathrm{G}_{m}\right), i=1,2$. In order to get the most effective bounds we need a slight generalization of the usual exponential sequence. So suppose $j: Y_{0} \subset Y$ is a closed subscheme defined by an ideal $I$ such that $I^{p}=0$. Then there is an exact sequence of sheaves in the Zariski (or fppf) site on $Y$,

$$
0 \rightarrow I \stackrel{e}{\rightarrow} \mathbf{G}_{m, Y} \rightarrow j_{*} \mathbf{G}_{m, Y_{0}} \rightarrow 0
$$

where $e$ is defined by

$$
e(g)=\sum_{0}^{p-1} g^{n} / n !, \quad g \in \Gamma(U, h) .
$$

Since $g^{i} h^{p-i}=0$ for $g, h \in \Gamma(U, I), e$ is a homomorphism with log as an inverse. In particular the kernels of $\operatorname{Pic}(Y) \rightarrow \operatorname{Pic}\left(Y_{0}\right)$ and $\operatorname{Br}^{\prime}(Y) \rightarrow \operatorname{Br}^{\prime}\left(Y_{0}\right)$ are $p$-torsion.

If $Y \subset X$, we say that the algebraic codimension of $Y$ in $X$ is less than or equal to $d$ if $O_{Y, y}$ is the quotient of $O_{X, y}$ by an ideal generated by less than or equal to $d$ elements for all $y \in Y$. Thus if $X$ is regular and $Y$ is a local complete intersection, then the algebraic codimension coincides with the geometric codimension of $Y$ in $X$.

THEOREM. Let $i: Y \rightarrow \mathrm{P}^{m}$ be a subvariety of $\mathrm{P}^{m}$ of algebraic codimension $d$ with $H^{1}\left(Y, O_{Y}(-1)\right)=0$. Let $t$ be any integer greater than $\log _{p}(d)$.

(1) Suppose $H^{1}\left(Y, I^{n} / I^{n+1}\right)=0$ for $n \gg 0$. Then $\operatorname{Pic}(Y)(p)$, the p-pri- 
mary component of $\operatorname{Pic}(Y)$, is a p-group of exponent no more than $p^{t}$.

(2) Suppose $H^{i}\left(Y, I^{n} / I^{n+1}\right)=0$ for $n \gg 0$ and $i=1$, 2. Then $\operatorname{Pic}(Y)$ is an extension of a finite group whose p-primary component has exponent at most $p^{t}$ by $Z\left[O_{Y}(1)\right]$.

(3) Suppose $H^{2}\left(Y, I^{n} / I^{n+1}\right)=0$ for $n \gg 0$. Then $\operatorname{Br}^{\prime}(Y)(p)$, the p-primary component of $\operatorname{Br}^{\prime}(Y)$, is a group of exponent no more than $p^{t}$ which is finite if $Y$ is smooth and $k$ is finite.

Proof. $t$ enters into the calculation in the following manner. Let $r \gg 0$. Then $I^{p^{t} \cdot p^{r}} \subset I^{(r)} \subset I^{p^{r}}$ and so the nilpotent embedding, $Y_{p^{r}} \hookrightarrow Y_{(r)}$, may be factored into a sequence of $t$ nilpotent embeddings each defined by an ideal $I_{k}$ with $I_{k}^{p}=0$,

$$
Y_{p r}=\tilde{Y}_{0} \hookrightarrow \tilde{Y}_{1} \hookrightarrow \cdots \hookrightarrow \tilde{Y}_{t-1} \hookrightarrow Y_{(r)},
$$

corresponding to the chain

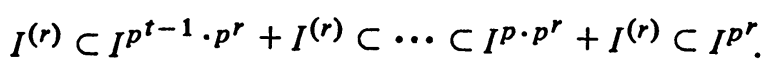

The exponential sequence then shows that the kernels of $\operatorname{Pic}\left(Y_{(r)}\right) \rightarrow \operatorname{Pic}\left(Y_{p^{r}}\right)$ and $\operatorname{Br}^{\prime}\left(Y_{(r)}\right) \rightarrow \operatorname{Br}^{\prime}\left(Y_{p}\right)$ are $p$-groups of exponent at most $p^{t}$.

(1) Since $H^{1}\left(Y, I^{n} / I^{n+1}\right)=0$ for $n \gg 0$, the exponential sequence

$$
0 \rightarrow I^{n} / I^{n+1} \rightarrow \mathrm{O}_{Y_{n+1}}^{*} \rightarrow \mathrm{O}_{Y_{n}}^{*} \rightarrow 0
$$

shows that $\operatorname{Pic}\left(Y_{n+1}\right) \rightarrow \operatorname{Pic}\left(Y_{n}\right)$ is injective for $n \gg 0$. Proposition 2 shows that $f^{* r}: \operatorname{Pic}(Y) \rightarrow \operatorname{Pic}\left(Y_{(r)}\right)$ is also injective. Consequently the factorization of $F_{Y(r)}^{s}$ through $f^{s}$ for sufficiently large $s$ and the argument above show that $\operatorname{Pic}(Y)(p)$ has exponent at most $p^{t}$.

(2) If $X \subset P^{m}$, let $P(X)=\operatorname{Pic}(X) / Z\left[O_{X}(1)\right]$. Proposition 2 shows that $f^{*}: P\left(Y_{(r)}\right) \rightarrow P\left(Y_{(r+1)}\right)$ is an isomorphism. As above the hypotheses and the exponential sequence shows that $\operatorname{Pic}\left(Y_{n+1}\right) \rightarrow \operatorname{Pic}\left(Y_{n}\right)$ is an isomorphism for $n \gg 0$. Thus, for $r, s \gg 0$, the exponential sequence, the remarks above, and the hypotheses show that $F_{Y}^{* s}$ factors as below:

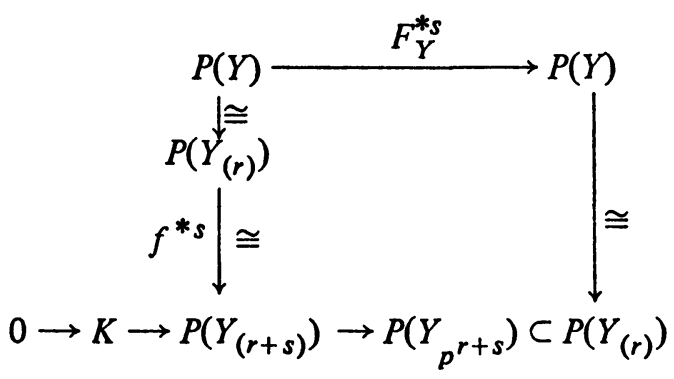

where the bottom row comes from the embedding $Y_{(r)} \subset Y_{p^{r+s}} \subset Y_{(r+s)}$ and $K$ is a $p$-group of exponent at most $p^{t}$. Since $F_{Y}^{* s}$ is multiplication by $p^{s}$ on $\operatorname{Pic}(Y)$, the definition of $P(Y)$ shows that $\operatorname{Pic}(Y)(p)=K$. Now $\operatorname{Pic}_{Y / k \text { red }}^{0}$ is a 
linear group since $\operatorname{Pic}(Y)(p)$ is $p^{t}$-torsion. Hence the torsion in $\operatorname{Pic}(Y)$ of order prime to $p$ is finitely generated as is $\operatorname{Pic}(Y) / \operatorname{Pic}(Y)_{\text {tors }}$. Since the cokernel of $F_{Y}^{* s}$ is $p^{t}$-torsion, the rank of $\operatorname{Pic}(Y)=1$ and $\operatorname{Pic}(Y) / \operatorname{Pic}(Y)_{\text {tors }}$ is generated by $O_{Y}(1)$ or $L$ where $L^{\otimes p^{i}} \cong \mathcal{O}_{Y}(1)$ for $i \leqslant t$ which completes the desired decomposition.

(3) The exponential sequence in the fppf topology and the hypothesis show that $\operatorname{Br}^{\prime}\left(Y_{n}\right) \rightarrow \operatorname{Br}^{\prime}\left(Y_{n-1}\right)$ is a monomorphism if $n \gg 0$. On the other hand Proposition 2 shows that $f^{*}: \operatorname{Br}^{\prime}\left(Y_{(r)}\right) \rightarrow \operatorname{Br}^{\prime}\left(Y_{(r+1)}\right)$ is injective for all $r$. Since $F_{Y}^{*}: \operatorname{Br}^{\prime}(Y) \rightarrow \operatorname{Br}^{\prime}(Y)$ is multiplication by $p$, factoring $F_{Y}^{* s}$ and arguing as above shows that $\operatorname{Br}^{\prime}(Y)(p)$ is at most $p^{t}$-torsion. If $Y$ is smooth, then the kernel of $F_{Y}^{*}$ can be computed by [3]. In particular it is an extension of a finite $p$ group by a vector space over $k$ and so is finite if $k$ is finite.

Our first corollary was suggested by Spencer Bloch.

Corollary 1. Let $Y=\mathbf{P}^{r_{1}} \times \mathbf{P}^{r_{2}} \rightarrow \mathbf{P}^{m}$ be the Segré embedding defined by an ideal $I$. Then $H^{2}\left(Y, I^{n} / I^{n+1}\right)$ is not always zero for large $n$.

Proof. Since $H^{1}\left(Y, O_{Y}(-1)\right)=0$ by the Künneth formula and the rank of $\operatorname{Pic}(Y)>1$, either $H^{1}\left(Y, I^{n} / I^{n+1}\right)$ or $H^{2}\left(Y, I^{n} / I^{n+1}\right)$ satisfies the corollary. Let $p_{i}: Y \rightarrow \mathrm{P}^{r_{i}}$ be the respective projection maps. Then there is an exact diagram

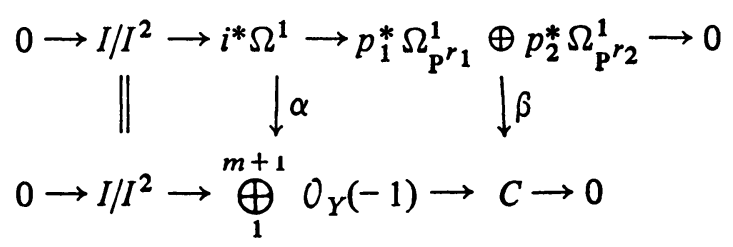

and cokernel $\beta=$ cokernel $\alpha=O_{Y}$. This immediately shows that $H^{1}\left(Y, I / I^{2}\right)=0$ and $H^{2}\left(Y, I / I^{2}\right) \neq 0$. Now $S^{n}\left(I / I^{2}\right)=I^{n} / I^{n+1}$ where $S^{n}$ denotes the $n$th symmetric power, and $S^{n}\left(\bigoplus_{1}^{m+1} O_{Y}(-1)\right)$ has a descending filtration whose successive quotients are $I^{j} / I^{j+1} \otimes S^{n-j}(C)$. But $H^{1}\left(Y, S^{n}\left(\bigoplus_{1}^{m+1} O_{Y}(-1)\right)\right)=0$ and so the vanishing of $H^{1}\left(Y, I^{n} / I^{n+1}\right)$ will follow from proving that

$$
H^{0}\left(Y,\left(I^{j} / I^{j+1}\right) \otimes S^{n-j}(C)\right)=0
$$

for $j<n$. But $S^{n-j}(C)$ has a filtration whose successive quotients are

$$
S^{k}\left(p_{1}^{*} \Omega_{\mathrm{P}^{r_{1}}}^{1} \oplus p_{2}^{*} \Omega_{\mathrm{p}^{r_{2}}}^{1}\right)
$$

for $k \leqslant n-j$. Since $S^{k}\left(p_{i}^{*} \Omega_{\mathrm{p}^{r_{i}}}^{1}\right)$ is contained in $p_{i}^{*}\left(S^{k}\left(\bigoplus_{1}^{r_{1}+1} O_{\mathrm{p}^{r_{i}}}(-1)\right)\right)$, the desired assertion follows immediately.

COROLlary 2. Let $i: Y \rightarrow \mathrm{P}^{m}$ be a smooth subvariety defined over a finite field $\mathbf{F}_{q}$ with $H^{1}\left(Y, O_{Y}(-1)\right)=0$ and $H^{2}\left(Y, I^{n} / I^{n+1}\right)=0$ for $n \gg 0$. Suppose $q=p^{r}$ with $p \neq 0,2$ and $\operatorname{dim} Y<p$. Then the rank of the group of $k$-rational points on the Picard scheme of $Y$ equals the order of the pole of $\zeta(Y, s)$ 
at $s=2 ;$ that is, the Birch-Tate-Swinnerton Dyer conjecture holds for cycles of codimension 1.

Proof. Bloch has recently shown that

$$
H^{2}\left(Y_{\mathrm{pl}}, Q_{p}(1)\right)=\left(\lim _{\longleftarrow} H^{2}\left(Y_{\mathrm{pl}}, \mu_{p^{n}}\right)\right) \otimes \mathbf{Q}
$$

is isomorphic to the subspace of $H_{\text {cris }}^{2}(Y / W) \otimes K$ on which the Frobenius acts as multiplication by $p$ [1]. Consequently if $q=p^{r}, F_{Y}^{r}: Y \rightarrow Y$ acts via multiplication by $q$ on $H_{\text {cris }}^{2}(Y / W) \otimes K$. But the dimension of this eigenspace is the order of the pole of $\zeta(Y, s)$ at $s=2$ [4]. Consequently the Kummer sequence reduces the Birch-Tate-Swinnerton Dyer conjecture to proving that $\operatorname{Br}^{\prime}(Y)(p)$ is finite.

Finally we need some examples where the hypotheses are satisfied. Short of proving the Kodaira vanishing theorem in char $p$, there are two ways $H^{1}\left(Y, O_{Y}(-1)\right)=0$ if $\operatorname{dim} Y=d \geqslant 2$.

(1) $H^{1}\left(Y, O_{Y}\right)=0 \Rightarrow H^{1}\left(Y, O_{Y}(-1)\right)=0$ : Bertini's theorem and the short exact sequence defining the structure sheaf of $D$, a generic hyperplane section of $Y$, shows that $H^{1}\left(Y, O_{Y}(-1)\right)=0$ if $Y$ is a variety.

(2) $Y$ smooth of dimension $\geqslant 3, \quad \operatorname{Pic}_{Y / k}^{0}$ has maximal $p$-rank $\Rightarrow$ $H^{1}\left(Y, O_{Y}(-1)\right)=0$ : We may assume $k$ is algebraically closed. The short exact sequence

$$
0 \rightarrow H^{1}\left(Y_{\mathrm{et}}, Z / p Z\right) \rightarrow H^{1}\left(Y, \mathrm{O}_{Y}\right) \stackrel{F_{Y}^{*}-I^{*}}{\longrightarrow} H^{1}\left(Y, \mathrm{O}_{Y}\right) \rightarrow 0
$$

and the assumption show that $H^{1}\left(Y, O_{Y}\right)$ has a basis $\left\{e_{i}\right\}$ for which $F^{*}\left(e_{i}\right)=e_{i}$. Now Bertini's theorem for $\pi_{1}$ shows that $H^{1}\left(Y, O_{Y}\right) \rightarrow H^{1}\left(D, O_{D}\right)$ is an isomorphism if $D$ is a generic hyperplane section, and so as above $H^{1}\left(Y, O_{Y}(-1)\right)=0$.

Recall that $\Gamma^{n}(E)$ is defined to be $\left(S^{n}\left(E^{\bigvee}\right)\right)^{\vee}$ for any vector bundle $E$ on $Y$ where $S^{n}$ is the $n$th symmetric power. In the terminology of [2],

$$
H^{i}\left(Y, \Gamma^{n}(E) \otimes F\right)=0
$$

for $n \gg 0$, any coherent sheaf $F$, and $i>0$ means $E$ is $\Gamma$-ample. Now in char 0 , $\Gamma^{n}(E) \cong S^{n}(E)$ and so $\Gamma$-ample and ample coincide. Serre duality and the ampleness of the normal bundle for $Y \subset \mathrm{P}^{m}$ show that $H^{d-i}\left(Y, \Gamma^{n}\left(N_{Y / \mathrm{P} m}\right) \otimes \omega\right) \cong$ $H^{i}\left(Y, I^{n} / I^{n+1}\right)=0$ for $n \gg 0$ and $i \neq d$. In char $p$ this is, of course, false in general. However $\Gamma^{n}(E) \cong S^{n}(E)$ if $E$ is a sum of line bundles and so it holds for strict complete intersections of dimension $>2$. By using base change we can "spread this out" to a larger class of examples.

Consider the following situation: $S$ is a variety over $k, i: Y \rightarrow \mathrm{P}_{S}^{m}$ is a regular embedding defined by an ideal $I$ such that the fibres of the natural map $q$ : $Y \rightarrow S$ are varieties of dimension $d>2$, and suppose that for some closed point $s_{0} \in S, Y_{s_{0}} \rightarrow \mathrm{P}^{m}$ satisfies the hypotheses of the theorem. Then for $n \gg 0$, $H^{d}\left(Y_{t}, \Gamma^{n}\left(N_{Y_{t} / \mathrm{P} m}\right) \otimes \omega_{Y_{t}}\right)=0$ for any $t \in S$ and so 


$$
R^{d} q_{*}\left(\Gamma^{n}\left(N_{Y / P_{S}^{m}}\right) \otimes \omega_{Y / S}\right)=0
$$

Moreover by Grothendieck's base change yoga there is an open set $U \subset S$ containing $s_{0}$ such that

$$
H^{i}\left(Y_{t}, I_{t}^{n} / I_{t}^{n+1}\right) \cong H^{d-i}\left(Y_{t}, \Gamma^{n}\left(N_{Y_{t}}\right) \otimes \omega_{Y_{t}}\right)=0
$$

for all $t \in U, n \gg 0, i=1,2$. Shrinking $U$ somewhat if necessary we can also ensure that $H^{1}\left(Y_{t}, O_{Y_{t}}(-1)\right)=0$ for $t \in U$. Thus if the hypotheses hold for $Y \subset \mathrm{P}^{m}$ then they hold for the generic deformation of $Y$.

\section{BIBLIOGRAPHY}

1. S. Bloch, Algebraic K-theory and crystalline cohomology (to appear).

2. R. Hartshorne, Ample subvarieties of algebraic varieties, Lecture Notes in Math., vol. 156, Springer-Verlag, Berlin and New York, 1970.

3. R. Hoobler, Cohomology of purely inseparable Galois coverings, J. Reine Angew. Math. 266 (1974), 183-199.

4. J. Tate, Algebraic cycles and poles of zeta functions, Arithmetical Algebraic Geometry (Proc. Conf. Purdue Univ., 1963), Harper and Row, New York, 1965, pp. 93-110. MR 37 \#1371.

DEPARTMENT OF MATHEMATICS, RICE UNIVERSITY, HOUSTON, TEXAS 77001 York 10031

Current address: Department of Mathematics, City College (CUNY), New York, New 